\title{
AMÉRICA LATINA: regímenes de bienestar en transición durante los años noventa ${ }^{1}$
}

\author{
Carlos Barba Solano*
}

\section{INTRODUCCIÓN}

En América Latina, la crisis económica de 1982 redefinió el escenario para la intervención estatal y consolidó a nuevos actores globales y locales. Estos pusieron en marcha una agenda económica y otra social radicalmente distintas a las que caracterizaron a la etapa de industrialización orientada al mercado interno.

La nueva bitácora económica fue sintetizada inicialmente en el "Consenso de Washington", el cual promovía el libre mercado, políticas macroeconómicas prudentes y la apertura comercial, a través de una serie de reformas estructurales encaminadas a: corregir los desequilibrios fiscal y externo, privatizar los bienes y servicios públicos, liberalizar el comercio, desregular el mercado laboral y financiero, y reformar los sistemas tributario y de pensiones.

Por su parte, la agenda social fue establecida

* Professor-investigador Titular da Universidade de Guadalajara. Centro Universitário de Ciencias Sociales y Humanidades. Av. Alcalde y de los Maestros, Sector Hidalgo. Guadalajara-Jalisco - México. Cep: 44260. cabarba@cybercable.net.mx

${ }^{1}$ Este trabajo está basado, en buena medida, en Barba (2006). gradualmente por el Banco Mundial (BM) y el Banco Interamericano de Desarrollo (BID) a través una serie de recomendaciones para enfrentar la pobreza, sin poner en tela de juicio el funcionamiento del mercado. Esas indicaciones han llegado a conformar un nuevo paradigma de bienestar para la región, que se plantea como un centro de confluencia discursiva y práctica para todos los países. Se ha marcado una ruta alternativa, que ha llegado a ser hegemónica, para abordar la cuestión social y se ha intentado poner en sintonía los sistemas de protección social regionales con los procesos de estabilización y ajuste económico.

A pesar de las enormes diferencias geográficas, sociales, demográficas y económicas entre los países que conforman la región, durante los años noventa la mayoría de los gobiernos de la región estuvieron de acuerdo ${ }^{2}$ con estas agendas. Sin embargo, el cambio paradigmático no ha redundado en una clara mejoría del bienestar en América Latina, ni siquiera en etapas de crecimiento, como la de la década de $1990 .^{3}$

\footnotetext{
${ }^{2}$ No todos, ni en los mismos grados, como lo veremos. ${ }^{3}$ De hecho, esos años han sido catalogados por la Comisión Económica para América Latina (CEPAL) como de "luces
} 
Frente a los magros logros alcanzados, tanto el consenso original como el patrón de reformas se han desgastado, dando pie a la aparición de conceptos, lecturas y propuestas diferentes respecto a lo qué debe considerarse prioritario en términos económicos y sociales a escala regional o nacional. ${ }^{4}$ Esto ha llevado a un cuestionamiento del paradigma de bienestar residual en el ámbito intelectual, a cambios de orientación en varios gobiernos $^{5}$ y a nuevas propuestas por parte de algunas agencias internacionales ligadas al tema del desarrollo, particularmente en el marco de las Naciones Unidas. ${ }^{6}$

En ese contexto, a partir de una perspectiva comparativa, este trabajo se propone dos cosas: analizar distintas trayectorias de los procesos de ajuste económico y reforma social en tres conjuntos de países y confrontar la tesis de que la residualización de todos los regímenes de bienestar latinoamericanos continúa siendo la única ruta posible.

Para realizar esta tarea, este trabajo abordará tres tópicos: (1) las características del paradigma de bienestar residual hegemónico en la región a partir de los años noventa; (2) la pertinencia de adoptar un enfoque comparativo para realizar análisis empíricos sobre esta temática y el uso del concepto de régimen de bienestar en América Latina; y (3) el análisis de los datos sociales disponibles, agrupados alrededor de tres tipos distintos de regímenes de bienestar regionales.

y sombras”, precisamente porque, a pesar de que durante varios años se reestableció el crecimiento económico los resultados sociales fueron desalentadores. (CEPAL, 2001) De acuerdo con datos generados por la CEPAL los países de la región no sólo continúan enfrentando hoy serios retos en términos de persistencia de procesos de exclusión, pobreza y alta vulnerabilidad social, sino también crecientes problemas de desempleo, subempleo, informalidad, desigualdad social y de género, e inseguridad ciudadana. (CEPAL, 2001; CEPAL/UNICEF, 2001).

${ }^{4}$ Ver Barba (2004) y Barba, [et al.] (2005).

${ }^{5}$ En el ámbito gubernamental el "giro a la izquierda" de los gobiernos de Venezuela, Chile, Brasil, Argentina y recientemente Uruguay así lo indican.

${ }^{6}$ Tal es el caso de PNUD, CEPAL e IINUPDS.

\section{ELPARADIGMARESIDUALENAMÉRICALATINA}

En América Latina, en el terreno social, de manera muy general, puede hablarse tanto del abandono paulatino del paradigma del seguro social, que tenía como eje al empleo formal y los derechos sociales que se adquirían por esa vía, como de la reducción progresiva de lo social a los temas de la pobreza y la vulnerabilidad social.

La transición ha implicado que, en la agenda de muchos de los gobiernos de la región, el tema de la ciudadanía social haya sido desechado o que se hayan interrumpido las acciones consistentes y suficientes para asegurar la universalización de derechos sociales.

Este camino ha conducido tanto a que el empleo sea concebido estrictamente como un problema de mercado, y por ello las tendencias más marcadas en ese ámbito sean la desregulación y flexibilización de los mercados de trabajo, como al imperativo de reformar de los sistemas pensionarios que, en muchos casos, han pasado de un modelo de reparto a otro de capitalización individual. ${ }^{7}$

La pauta dominante ha sido la residualización de la acción pública en materia social, esto es: asumir que las políticas y programas sociales deben dirigirse exclusivamente a quienes son incapaces de contratar su propia previsión social en términos privados.

La idea más fuerte ha sido que el papel de la política social debe restringirse a garantizar a los más pobres recursos mínimos para que cada uno pueda aprovechar las oportunidades de ingreso que supuestamente el mercado proporciona. Este enfoque, que concibe la pobreza como un problema imputable fundamentalmente a deficiencias de carácter individual, ha servido para justificar el carácter focalizado de los programas sociales,

\footnotetext{
Aunque este proceso ha sido sumamente azaroso, podría decirse que ni las recetas del Banco Mundial (1994) ni la experiencia chilena, que ha servido de modelo en el proceso de reforma, han sido seguidas a pie juntillas, en buena medida porque internamente los gobiernos reformistas han enfrentado distintos grados de oposición. Esto ha dado pie a la aparición de tres modelos distintos, además del ya existente: el sustitutivo, el mixto y el paralelo. Para una exposición detallada sobre este tema ver: Barba (2003, p. 278-296)
} 
frecuentemente pensados en oposición a políticas universalistas. Desde esa óptica, sólo se considera legítimo reasignar recursos por medios públicos a quienes, además de situarse en la pobreza extrema, están dispuestos a alcanzar su bienestar jugando de acuerdo con las reglas del mercado.

Esa ha sido la racionalidad dominante en el diseño de fondos de inversión social o de programas orientados tanto a la acumulación de capital humano, como a garantizar activos mínimos o establecer redes de seguridad que operen en situaciones críticas.

Generalmente, la población objetivo de esta clase de intervenciones ha sido: quienes viven en pobreza extrema, particularmente las nuevas generaciones de pobres ${ }^{8} \mathrm{y}$ aquellos considerados más vulnerables a las crisis económicas, las catástrofes naturales o familiares, que corren el riesgo de caer por debajo de las líneas de pobreza establecidas.

El paradigma naciente, encabezado por el BM y el BID, admitido y promovido devotamente por numerosos gobiernos latinoamericanos, se distingue por su perfil trasnacional, pues supone la participación de actores internacionales en un tema que tradicionalmente había sido competencia exclusiva de los estados nacionales: el bienestar social. Por ello podríamos hablar de la deslocalización como una de sus primeras características; ${ }^{9}$ la otra sería, por supuesto, el respeto a la libertad de mercado y el atribuirle a éste el papel fundamental en la generación y distribución de bienestar, ${ }^{10}$ aspec-

${ }^{8}$ La intención ha sido interrumpir la reproducción intergeneracional de la pobreza, aunque en México se habla ya de tres bandas de pobreza: la alimentaria, la de capacidades y la patrimonial. En teoría, cada una de ellas demandaría distintos tipos de acciones públicas: transferencias incondicionales, programas de capital humano, programas de dotación de activos. (Cortés, [et al.], 2002). Las dos primeras corresponderían, a grosso modo, a las visiones tradicionales sobre pobreza extrema y la tercera a la concepción de vulnerabilidad social.

${ }^{9}$ La deslocalización no se refiere exclusivamente a un cambio de escala, sino a la adquisición de capacidades que antes eran predominantemente estatales por parte de estos nuevos actores, tales como: el diseño, el financiamiento y la evaluación de programas sociales. Por supuesto, esto no significa que las agencias internacionales monopolicen dichas funciones, sino que ahora son actores cruciales en cada una de ellas.

${ }^{10}$ Lo que se expresa en la tendencia a considerar el crecimiento económico como el mecanismo fundamental para reducir la pobreza. to que le confiere un marcado carácter residual. ${ }^{11}$

\section{LA HETEROGENEIDAD REGIONAL Y LA CONCEPTUALIZACIÓN DE LO SOCIAL}

Suele decirse que, en América Latina, el "modelo de bienestar" ha sido semejante al Estado social conservador o corporativo europeo y que paulatinamente, tras más de una década de reformas económicas y sociales, se ha ido acercando al modelo residual anglosajón.

Ambas afirmaciones, aunque acertadas desde una perspectiva muy general, son reduccionistas, porque parten, al menos, de tres premisas inadecuadas: (1) que pueden aplicarse de manera mecánica las tipologías de los regímenes de bienestar de las economías desarrolladas a la realidad latinoamericana; (2) que puede hablarse de esta región como si fuera una realidad homogénea; y (3) que la aplicación de agendas de reforma económica y social semejantes produce los mismos resultados en todos los casos.

Para empezar, no es apropiado hablar en general de la existencia y continuidad de estados del bienestar en América Latina, donde, con muy escasas excepciones, no han prevalecido de manera duradera la democracia, ni se han garantizado todos los derechos civiles o políticos, o se han universalizado los derechos sociales para toda la población, ni se han alcanzado los niveles de salarización logrados por las grandes economías de Europa.

${ }^{11}$ Basados en experiencias históricas de países anglosajones como Estados Unidos, Australia, Canadá o Nueva Zelanda y tardíamente el Reino Unido, sabemos que los regímenes de bienestar de tipo residual asumen que el mecanismo fundamental para alcanzar el bienestar social es el mercado y que las prestaciones propiamente públicas se deben orientar sólo a corregir externalidades de la economía, asignando recursos a los más pobres, para que sean capaces de participar en el mercado y sobreponerse por sí mismos a sus dificultades (Skocpol, 1995, p. 7; Hill; Bramley, 1986, p. 10). Las normas para la asistencia social son estrictas, frecuentemente asociadas con procesos de estigmatización, y los beneficios suelen ser modestos, ya que se piensa que los beneficios excesivos reducen la motivación para trabajar (Esping-Andersen, 1990, p. 26; EspingAndersen; Corpi, 1993, p. 370, 373). El enfoque residual de la política social tiende a respetar la estratificación social generada por el libre curso de la oferta y la demanda, por lo que sus efectos desmercantilizadores son mínimos. Ibid., p. 372 . 
Tampoco es adecuado abordar a la región como un todo, porque, evidentemente, existen grandes diferencias entre los países que la integran. Los contrastes se manifiestan en varias escalas, como: la territorial, la demográfica, la etno-cultural, la de las dimensiones del producto interno bruto, la de los procesos de industrialización, etc. ${ }^{12}$ Las discrepancias son particularmente agudas en el terreno del bienestar social en aspectos como: los niveles de gasto social, ${ }^{13}$ los niveles de desarrollo de los sistemas de prestaciones sociales, ${ }^{14}$ los grados de madurez institucional, ${ }^{15}$ las tendencias en materia de ampliación de cobertura, ${ }^{16}$ los grados de exclusión social, ${ }^{17}$ las trayectorias en materia de distribución del ingreso, ${ }^{18}$ los niveles de pobreza

${ }^{12}$ Para un recuento detallado de estas diferencias, ver Barba (2003, p. 420-426).

${ }^{13}$ Existen enormes disparidades en materia de gasto social en la región, entre aquellos países que, como Uruguay, Argentina, Costa Rica, Chile o Brasil, hacia 1980-1981 gastaban como promedio el 16\% del PIB, mientras países como Venezuela, Ecuador, Nicaragua, México y Colombia gastaban el $10.1 \%$, promedio muy cercano a promedio regional, y otros como Perú, Paraguay, Bolivia, Guatemala y Honduras gastaban menos de la tercera parte que los primeros y la mitad que los segundos, pues en promedio desembolsaban el 5\% del PIB. Los caso extremos son Chile y Paraguay pues existía entre los dos una diferencia de 15.6 puntos porcentuales (Ver: Barba 2003, p. 428-429; Cominetti; Ruiz, 1998).

${ }^{14}$ Este tipo de sistemas, ligados al modelo ISI, fueron muy significativos en países como Argentina, Brasil, Chile, Colombia, Costa Rica, México y Uruguay; en otros casos, como Guatemala, Paraguay, Perú, Ecuador, etc., fueron precarios o prácticamente inexistentes (Raczynski, 1999 p. 172; Filgueira, 1997, p. 83). Además, mientras países como Brasil y México no fueron más allá del sistema del seguro social limitado, otros como Argentina, Chile, Costa Rica y Uruguay crearon sistemas de protección socia universales (Malloy, 1986, p. 41).

${ }^{15}$ No todos los sistemas de prestaciones sociales en América Latina fueron creados al mismo tiempo; de hecho, pueden clasificarse en función del momento cuando fueron creados, como: pioneros (años veinte), intermedios (años treinta y cuarenta) y tardíos (años cincuenta en adelante) (Mesa-Lago, 1994). Esto supone diversos grados de maduración institucional. En el caso de los sistemas pioneros (Argentina, Brasil, Chile y Uruguay), encontramos que, en tres de ellos, se pueden detectar grados muy significativos de cobertura de algunas prestaciones sociales; mientras en algunos de los casos intermedios, encontramos avances mucho menos significativos, como ocurre en el caso de México.

${ }^{16}$ Mientras países como Argentina, Brasil, México, Uruguay y Chile siguieron una vía bistmarckiana de expansión gradual, Costa Rica siguió una vía beverdigeana de cobertura universal en un solo momento, y Cuba una vía socialista que, entre los años sesenta y setenta, completó un sistema de seguridad social universal- (Barba, 2003, p. 430).

17 Todo indica que, en América Latina, las sociedades con mayor heterogeneidad etno-cultural han tolerado mayores grados de exclusión social (Barba, 2003; p. 430-432).

${ }^{18}$ Esto puede apreciarse, por ejemplo, en los años setenta, cuando los Coeficientes de Gini a nivel urbano fluctuaban prevalecientes, ${ }^{19}$ los índices relativos de niveles de vida ${ }^{20}$ etc.

Por tanto, es incorrecto suponer que, a pesar de la heterogeneidad reinante en la región, la aplicación de agendas semejantes de reforma económica y social en distintos casos producirá resultados similares. Sin embargo, dada la gran heterogeneidad reinante y a pesar de las peculiaridades sociales de América Latina, la utilización del concepto de "regímenes de bienestar" es muy pertinente, precisamente para desentrañar el carácter diferencial de la construcción de lo social en distintos conjuntos de países. Pero, para ello, hace falta evitar una aplicación ontológica de ese concepto.

\section{LA GENEALOGÍA DEL CONCEPTO DE REGÍMES DE BIENESTAR}

A partir de la obra de Gosta EspingAndersen (1987, 1990, 1996, 1999, 2001), en el marco de la Organización para la Cooperación y el Desarrollo Económicos (OCDE), se ha desarrollado una intensa agenda de investigación social fundada en la comparación empírica de la calidad de los derechos sociales, la estratificación social y las estructuras de los mercados laborales de los países que la integran (Vg. Goodin, et al. 1999; Deacon, 1999; Bonoli; Taylor-Gooby, 2000; Scharpf; Schmid, 2000; Annesley, 2001; Huber; Stephens; Schierup, 2001).

El eje teórico-metodológico de dichos estudios ha sido el concepto de "régimen de bienestar", definido por Esping-Andersen como "la manera combinada e interdependiente como el bienestar es producido y asignado por el estado, el mercado y la familia.” (Esping-Andersen, 1999, p. 34-35). Donde "una organización dada de las relaciones entre [estas tres instancias] ... se asocia

entre 0.43 en el caso de Uruguay y 0.59 en el de Brasil, ver: Altimir (1995, Cuadro 2.1).

${ }^{19}$ Para una comparación de datos de pobreza urbana, rural y total en 10 países de América Latina durante los años setenta y ochenta, ver Altimir (Cuadro 2.3).

${ }^{20}$ Ver: Astorga; FitzGerald (1998, Cuadro IX.5). 
con una lógica particular de la política social...” (Esping-Andersen, 1987, p. 6-7).

Entre los múltiples resultados de los estudios mencionados, es conveniente rescatar dos que son muy adecuados para lo que aquí se argumenta: (1) la posibilidad y pertinencia de construir tipos ideales de arreglos entre las instancias mencionadas para recoger la variabilidad histórica de "lo social", ${ }^{21}$ (2) el gran potencial del concepto de "regímenes de bienestar" para realizar estudios sobre las transformaciones de los sistemas concretos de bienestar, las políticas sociales, los mercados laborales y las estrategias familiares en el contexto de la globalización y los procesos de integración económica.

Por lo que corresponde al primer punto, Esping-Andersen, en su libro sobre los tres mundos del bienestar en el capitalismo (1990), ha intentado demostrar que la mayoría de los estados del bienestar de la OCDE se agrupan (cluster) alrededor de tres tipos de regímenes de bienestar: el liberal o residual, el conservador o corporativo y el institucional o socialdemócrata. ${ }^{22}$

21 "Lo social" es entendido no como un lugar distinto a "lo económico" y "lo político", tampoco como un conjunto de instituciones paraestatales, sino como un conjunto de mediaciones entre tres órdenes: el económico, el político y el familiar (Lautier, 2001, p. 3; Barba, 2003).

${ }^{22}$ El liberal o residual, basado en experiencias históricas de países anglosajones como Estados Unidos, Australia, Canadá o Nueva Zelanda, asume que la mayoría de la población puede contratar su propia previsión social y que, por ello, el Estado sólo debe apoyar a aquel residual humano que es incapaz de velar por su propio bienestar, es decir, los más pobres (Skocpol, 1995; Hill; Bramley, 1986; Esping-Andersen, 1990; Esping-Andersen; Corpi, 1993). El conservador o corporativo, construido a partir de la experiencia histórica de los países de Europa Occidental Continental, pone al Estado y las instituciones públicas en el centro del proceso socioeconómico, los cuales distribuyen beneficios siguiendo criterios de clase o estatus político, los beneficios que no constituyen derechos universales, sino corporativos (aunque gradualmente se acercan a la universalización en condiciones de pleno empleo), tienden a reforzar la estratificación social que se deriva de las capacidades políticas de los actores y sólo se ofrecen cuando las capacidades de la familia para servir a sus miembros se han agotado (Esping-Andersen, 1990). El institucional o socialdemócrata suele ejemplificarse con dos experiencias históricas diferentes: el paradigma beveridgeano inglés y el social demócrata escandinavo, ambos desarrollados básicamente después de la Segunda Guerra Mundial. Cada uno de ellos concibe los riesgos como consecuencias sistémicas de la operación del mercado y, por ello, asumen el bienestar individual como una responsabilidad colectiva, solidaria. Ello explica que "lo social" incluya, en estos casos, al conjunto de los ciudadanos. Este enfoque implica la implantación del principio de universalidad de las políticas sociales, en
Por lo que toca al tema de los regímenes de bienestar en transición, el centro de los estudios aludidos ha sido demostrar empíricamente que no tienen fundamento tres tesis que son moneda de curso común en numerosos documentos generados por agencias financieras internacionales y en múltiples trabajos producidos bajo el paraguas del Consenso de Washington: (1) que el paradigma de bienestar residual es el único que se ajusta a las condiciones impuestas por la globalización y los procesos de integración económica; (2) que la única ruta posible es la desregulación laboral; y (3) que el Welfare State enfrenta una crisis terminal de la que ya no podrá recuperarse. ${ }^{23}$

\section{LOS REGÍMENES DE BIENESTAR LATINOAMERICANOS}

Más allá de la discusión de estas temáticas, las cuales he abordado en otros textos (Barba, 2003, 2004, 2004a), se encuentra una cuestión crucial para este trabajo: si el concepto de régimen de bienestar es apropiado para dar cuenta: (1) tanto de los arreglos institucionales desarrollados históricamente entre el Estado, la economía, las unidades domésticas y las políticas sociales en América Latina; (2) como del proceso y los alcances de la intensa agenda de reforma social que se ha operado a escala regional durante los años noventa.

En mi opinión, no hay ninguna razón para asumir que las únicas formas de articulación posibles entre los elementos referidos en el inciso

el caso beveridgeano, para fijar el derecho a un nivel mínimo de bienestar para todas las categorías y grupos sociales, encaminado a garantizar ciudadanía universal a pesar de las diferencias sociales generadas por la operación del mercado. En el caso socialdemócrata, a través de un proceso de desmercantilización sin precedentes en el marco de sociedades capitalistas, dirigido a construir un derecho democrático a un nivel de vida socialmente adecuado para construir una sociedad más equitativa, al margen de la lógica del mercado (Beveridge, 1987; EspingAndersen; Corpi, 1993; Hill, 1997; Lautier, 2001; Marshall, 1992; Torfing, 1998).

${ }^{23}$ Entre ellos, destacan los siguientes: Hill y Bramley 1986, Pierson 1994, Skocpol 1995, Goodin, et.al. 1999, Deacon 1999, Bonoli y Taylor-Gooby 2000, Scharpf y Schmid 2000, Esping-Andersen 1996, 1999, 2001, Annesley 2001, Huber y Stephens 2000, y Schierup 2001. 
"1" sean las que han prevalecido en los tipos de regímenes de bienestar de las grandes economías de la OCDE. En América Latina, las políticas sociales y los sistemas de prestaciones sociales tienen una larga historia. ${ }^{24}$ La fase expansiva de dichos sistemas estuvo acoplada con el proceso de industrialización, a través de la sustitución de importaciones (ISI), en auge entre los años cuarenta y setenta. ${ }^{25}$

Durante esos años, el sistema del seguro social fue uno de los ejes para articular coaliciones distributivas partidarias del proyecto industrializador, que estuvieron integradas por sectores de las clases medias, organizaciones obreras, empleados públicos, empresarios industriales nacionales y extranjeros, partidos políticos y funcionarios públicos.

De igual forma, es cierto que, en lo general, las instituciones del seguro social se distinguieron por ser regresivas y no democráticas, características que:

- sólo en pocos casos permitieron un proceso expansivo de derechos y ciudadanía social, lo que se tradujo en una situación de exclusión asistencial de quienes no formaban parte de la coalición que retroalimentaba el proyecto de industrialización, especialmente los trabajadores del sector informal urbano y quienes laboraban en el sector rural.

- y que propiciaron altos niveles de desigualdad en la distribución del ingreso, la cobertura de la protección social y la calidad de los servicios sociales $^{26}$ (Barba, 2003, p. 384-393).

Se puede agregar a esta descripción que esas

${ }^{24}$ Los sistemas de prestaciones sociales en la zona son de larga data, pues se han desarrollado en tres grandes oleadas, la primera durante los años veinte, la segunda inicia en los años cuarenta y la tercera a partir de los años cincuenta (Mesa-Lago, 1994). De hecho, como lo señala James Malloy, muchos países latinoamericanos adoptaron programas de seguridad social antes que países capitalistas industrializados como los EUA (Malloy, 1986, p. 31).

${ }^{25}$ Algo parecido a la relación que se produjo en Europa Occidental y los Estados Unidos entre la expansión de Estado del bienestar y las políticas keynesianas.

${ }^{26}$ En la región, la hegemonía de este paradigma fue acompañada con altos niveles de desigualdad, así como por una tendencia a la reducción del peso relativo de la pobreza, pero no de su peso absoluto. Sin embargo, los indicadores de calidad de vida muestran que, en la etapa expansiva del modelo ISI, mejoraron consistentemente los principales indicadores sociales, como las tasas de mortalidad infantil, la esperanza de vida y la cobertura de los sistemas de educación y salud (Barba, 2003). instituciones expresaban una concepción familiarista del bienestar social, pues preservaban una división familiar del trabajo (breadwinner system) que confería a los hombres adultos el papel de proveedores y portadores de derechos para el resto de la familia, a través del empleo formal, y a las mujeres la responsabilidad de transferir servicios de bienestar para los demás miembros del hogar (Barba, 2003).

Sin embargo, como lo he sostenido en un extenso estudio sobre los sistemas de bienestar en Latinoamérica (2003), no es correcto hablar de un solo tipo de régimen de bienestar en la región, sino de tres tipos desarrollados bajo el paraguas del paradigma del seguro social: los universalistas, los duales y los excluyentes.

Los regímenes universalistas, que incluían a Chile, Argentina, Uruguay y Costa Rica, ${ }^{27}$ fueron los que más se asemejaron a los regímenes conservadores europeos, tanto por su expansión gradual y universalizante, ${ }^{28}$ como por la vinculación de la protección social al mercado laboral formal y a las organizaciones de clase obrera. Sin embargo, no hubo uniformidad en los tipos de regímenes políticos con los que se vincularon, ya que estos se dividieron en democráticos y autoritarios. ${ }^{29}$

Los datos indican que, a inicios de los años setenta, cuando aún parecía viable el modelos ISI a escala latinoamericana, en estos países se observaban los mayores niveles de gasto social, la menor heterogeneidad etno-cultural, la mayor cobertura del seguro social y de los sistemas educativos y de salud, los menores niveles de pobreza rural, urbana y total; los menores índices de concentración del ingreso; los indicadores más bajos en materia de precariedad laboral y subempleo; los mejores indicadores en materia de esperanza de vida al nacer, mortalidad infantil, analfabetismo e índices relativos de vida (Barba, 2003, p. 444-464).

${ }^{27}$ Los tres primeros desarrollaron sus sistemas de bienestar desde principios del Siglo XX, mientras Costa Rica lo hizo a mediados del siglo pasado.

${ }^{28}$ La excepción fue Costa Rica, donde la cobertura se asemejó más al modelo Beveridgeano de cobertura universal en un solo momento.

${ }^{29}$ Sólo el caso costarricense se ha mantenido bajo un sistema político democrático, sin interrupciones autoritarias. 
Por su parte, los regímenes duales incluían a Brasil, México, Colombia y Venezuela, y los excluyentes a la mayoría de los países de América Central, con la excepción de Costa Rica y Panamá, así como a países de América del Sur como Ecuador, Perú, Bolivia y Paraguay.

Estos dos tipos de regímenes se distinguían por:

- un descenso de uno y dos escalones, respectivamente, en todos los indicadores ya mencionados, así como en los niveles de gasto social, de cobertura de seguridad social, de los servicios educativos y de salud preventiva, y del grado de desmercantilización del bienestar social.

- y un aumento gradual de la heterogeneidad etnocultural del carácter regresivo de los sistemas de protección social y del grado de informalidad de los mercados laborales. ${ }^{30}$

Estas características tendían a manifestarse de manera dual en los regímenes intermedios, privilegiando a la población urbana, organizada y relevante para el modelo ISI, y excluyendo al resto de la población, mientras se agudizaban en el caso de los regímenes excluyentes, dejando por fuera de su cobertura a la mayoría de la población (2003).

\section{REGÍMENES EN TRANSICIÓN}

Tras más de una década de reformas, los regímenes de bienestar latinoamericanos han sufrido transformaciones muy significativas. Los efectos de los procesos de ajuste económico y de reforma social, así como las dinámicas de los mercados laborales, se han distribuido de manera desigual entre los tres tipos de regímenes mencionados.

\section{a. Las características de los procesos de ajuste}

Las reformas no se iniciaron al mismo tiempo en toda la región. En los regímenes

\footnotetext{
${ }^{30}$ Para un recuento pormenorizado de esos indicadores, ver: Barba (2003).
}

universalistas del Cono Sur, las reformas iniciaron durante los años setenta, mientras en el resto de los países de América Latina la década de 1990 fue la más activa en esta materia, particularmente en su primera mitad (Barba, 2003, p. 546-550).

Por otra parte, los ritmos de las reformas no han sido similares; en regímenes universalistas como Chile, Uruguay y Costa Rica, al igual que en regímenes duales como Brasil y Colombia, las reformas ha tendido a ser graduales (lentas y crecientes), mientras en Argentina (régimen universalista), México, Venezuela (regímenes duales) y Perú junto con la mayor parte de Centro América (regímenes excluyentes) han abundado los tratamientos de shock (amplios y rápidos) (2003, p. 484-489).

Los alcances de los procesos de reforma tampoco son equivalentes; han abarcado numerosos campos, entre ellos la apertura comercial, la reforma tributaria, la reforma financiera, las privatizaciones de activos públicos, la reforma laboral y la reforma del sistema de pensiones. Sin embargo, puede documentarse una gran variabilidad en este terreno, pues mientras algunos países como Argentina, Chile (regímenes universalistas) o Perú (régimen excluyente) han realizado cinco o seis de estas grandes reformas, otros como Colombia, México, Venezuela (regímenes duales) y Bolivia, Guatemala o Nicaragua (regímenes excluyentes) han realizado cuatro, $\mathrm{y}$ otros más como Costa Rica, Uruguay (regímenes universalistas) o Brasil (régimen dual) han realizado dos o tres (Barba, 2003, p. 484-489).

Tampoco se puede hablar de homogeneidad en los años de crisis, corrección o estancamiento de esas reformas, o en el estado actual de las mismas. Las reformas tempranas en el Cono Sur entraron en crisis a principios de la década de 1980, las tardías entraron en crisis en los años noventa, como ocurrió en Brasil, México o Venezuela. La continuidad de las mismas ha sido igualmente variable: las reformas se han consolidado en Chile, han sido frenadas pero son estables en países como México, Brasil y Colombia, se encuentran en crisis y revisión en Argentina y han sido revertidas en Venezuela (2003, p. 484-489). 


\section{b. Los estilos de crecimiento y el empleo}

También en el caso de los estilos de crecimiento o las estrategias de empleo asumidas, podemos hablar de trayectorias diferentes que se ha traducido en dos distintas modalidades de exclusión laboral. La primera corresponde a los regímenes universalistas, que se caracterizan por una calidad del empleo relativamente alta, por altos niveles de desempleo y una alta concentración del ingreso. La segunda, que concierne tanto a los regímenes duales como los regímenes excluyentes, se caracteriza por niveles de desempleo intermedios, pero se destaca por la baja calidad de los nuevos empleos que genera, así como por la alta polarización salarial y por la concentración del ingreso que produce. ${ }^{31}$

\section{c. La reforma social}

La heterogeneidad reinante continúa en el caso de los procesos de reforma social. En este caso, como

31 Durante los años noventa, en los regímenes universalistas del Cono Sur, el crecimiento del empleo se concentró en sectores y subsectores que emplean trabajo calificado y asalariado (servicios básicos, los servicios financieros y los servicios sociales). Esos países tuvieron un crecimiento económico muy elevado (alrededor de 5.3\% anual), pero se distinguieron por una baja elasticidad empleo-producto (ya que, por cada punto porcentual de incremento del PIB, el empleo creció apenas 0.33 puntos porcentuales) y por muy altos niveles nas 0.33 puntos porcentuales) y por muy altos niveles
de desempleo urbano (tasas medias que fluctuaron entre 7.4 y $10,4 \%$ entre 1991 y 1999). Estas tendencias generaron una polarización salarial moderada y no permitieron la reducción de la concentración del ingreso (Barba, 2003, Cuadros 72 a 75; y Anexo II). Por su parte, en los regímenes duales, también crecieron los servicios básicos, financieros y sociales, pero esto fue acompañado por un crecimiento significativo del empleo en sectores y subsectores que emplean trabajo descalificado (particularmente en la industria manufacturera en el caso de México, en la industria de la construcción en el caso venezolano y el comercio en todos los casos). Estos países tuvieron un crecimiento económico medio (una tasa promedio de $2.9 \%$ anual), pero se distinguieron por una elasticidad empleo-producto mayor a la media regional (por cada punto porcentual de incremento del PIB el empleo creció $0.70 \%$ ), por altos niveles de desempleo urbano (las tasas medias anuales fluctuaron entre $6.8 \mathrm{y}$ 11.1 entre 1991 y 1999), por la baja calidad del empleo generado (gran peso del empleo por cuenta propia) y por una alta polarización salarial, que evitó la reducción de la concentración del ingreso (Barba, 2003, Cuadros 72 a 75; y Anexo II). Finalmente, regímenes excluyentes, como Bolivia, El Salvador, Honduras o Perú, se distinguieron por contar con el mayor crecimiento del empleo en sectores y subsectores que emplean trabajo descalificado de toda la región (el crecimiento del empleo lo afirma Filgueira (1998), la focalización y la descentralización no puede significar lo mismo en los regímenes universalistas donde se ha dado un desarrollo notablemente mayor de derechos universales en materia de educación y salud, que en el caso de los regímenes duales y excluyentes, que representan dos escalones inferiores en este terreno.

Si los procesos de focalización se enmarcan en derechos universales, pueden emplearse para garantizar un acceso mínimo a éstos a quienes no han sido incluidos y pueden contribuir a fortalecer la ciudadanía social. Sin embargo, existe el riesgo de usar esta estrategia para dejar en un segundo plano la ampliación de esta clase de ciudadanía, sobre todo en países donde los derechos universales no se han desarrollado significativamente, concentrándose retóricamente en la superación de la pobreza. Este riesgo crece en las zonas excluidas de los regímenes duales y en el conjunto de los regímenes excluyentes. En esos mismos casos, son mayores los riesgos de estigmatización social, exclusión asistencial y neoclientelismo.

Por lo que toca a la descentralización, más allá del carácter sumamente heterogéneo que ha adquirido este proceso en la región (Gropello; Cominetti, 1998), la clientelización de los servicios sociales y el neopatrimonialismo, al igual que la agudización de inequidades distributivas y la indisciplina fiscal, constituyen riesgos mayores en los regímenes duales y excluyentes, donde existen amplios sectores no incorporados a la protección social moderna (Barba, 2003, p. 554-556, 560-563).

en la industria manufacturera, en la de la construcción $\mathrm{y}$ en el comercio fue muy alto), tuvieron altas tasas de crecimiento del PIB (alrededor de $4.3 \%$ anual), se distinguieron por la mayor elasticidad empleo-producto de América Latina (por cada punto porcentual de incremento del PIB, el empleo creció $1.3 \%$ ), por niveles de desempleo urbano relativamente bajos (las tasas medias anuales que fluctuaron entre 6.8 y 7.7 entre 1991 y 1999), una baja calidad del empleo (empleo por cuenta propia) y una muy alta polarización salarial, atribuible al hecho de que también creció el empleo en los sectores que emplean trabajo calificado, lo que tendió a incrementar la concentración del ingreso (Barba, 2003, Cuadros 72 a 75; y Anexo II). 


\section{Las redes de protección social}

En ese mismo tenor, aunque en toda la región se ha promovido la creación de redes de seguridad ${ }^{32}$ para enfrentar la vulnerabilidad social a los ciclos económicos y las catástrofes naturales y personales, y para evitar estrategias familiares que implique costos a largo plazo en términos de desarrollo humano, ${ }^{33}$ las redes se han difundido a lo largo de América Latina de manera muy desigual.

En los regímenes universalistas, donde el problema del desempleo es mayor, han predominado las políticas de workfare, encaminadas a la reinserción laboral y a una cierta desfamiliarización del bienestar. ${ }^{34}$ En los regímenes duales, donde el problema de la pobreza es considerablemente mayor, destacan las transferencias de efectivo y la aparición de programas de desarrollo humano que tienen como eje el rol de reproductivo de las mujeres. ${ }^{35}$ Mientras en los regímenes excluyentes, donde la pobreza es muy severa, la característica distintiva son los fondos de inversión social (Barba, 2003, p. 563-577).

\section{La reforma de los sistemas pensionarios}

En el caso de la reforma de los sistemas de pensiones, nuevamente encontramos que aunque el modelo de reforma difundido en toda la región es el del sistema de capitalización individual

\footnotetext{
${ }^{32}$ De acuerdo con Hicks y Wodon, las características de una red de seguridad ideal serían las siguientes: estar basada en un análisis acabado de quiénes tienden a verse más afectados por la crisis y qué clase de mecanismos utilizan normalmente para enfrentarlas; ofrecer una cobertura suficiente a la población a la que se desea llegar, especialmente a los grupos más vulnerables y excluidos; estar bien focalizada en los pobres; hallarse bajo la supervisión de instituciones bien constituidas; ser anticíclicas; ser sustentables desde el punto de vista fiscal; poder entregar beneficios con rapidez y lograr que la mayor parte posible de los costos se dirijan a incrementos netos del ingreso de los beneficiarios; complementar los programas sociales privados; reducirse proporcionalmente una vez que pasa la crisis (Hicks; Wodon, 2001, p. 99-100).

33 Particularmente el trabajo infantil y juvenil, que sustituyen la escolaridad y se traducen en pérdidas salariales de largo plazo, debido a menores dotaciones de capital humano. Ibid, p.96.

${ }^{34}$ El apoyo de jóvenes y mujeres, la creación de empleos en las pequeñas y medianas empresas y los programas de capacitación para el empleo.

${ }^{35} \mathrm{Tal}$ es el caso de Progresa-Oportunidades en México.
}

desarrollado en Chile desde 1981, las reformas han tenido características dispares, debido sobre todo a la oposición política y social interna que han enfrentado las iniciativas de reforma. Se puede hablar de dos tipos de reforma: las autoritarias, ejemplificadas por los casos de Chile, México y Perú, y las democráticas, ilustradas por los casos de Argentina, Costa Rica, Uruguay y Colombia, que han generado cuatro tipos de sistemas y un nuevo tipo de programas emergente: el sustitutivo, ${ }^{36}$ el mixto, ${ }^{37}$ el paralelo, ${ }^{38}$ los sistemas públicos de reparto y capitalización colectiva reformados ${ }^{39} \mathrm{y}$ programas de pensiones no contributivas, como los existentes en Brasil..$^{40}$

\section{Los rendimientos de las reformas}

De acuerdo con datos aportados por diversas fuentes,$^{41}$ es posible establecer algunos patrones sobre los efectos que las reformas estructurales y sociales han tenido sobre las capacidades de los regímenes de bienestar latinoamericanos para producir y distribuir bienestar.

${ }^{36}$ En el que un sistema de capitalización individual reemplaza a un sistema de reparto o de capitalización colectiva. Este modelo sigue la experiencia chilena y sólo ha podido implantarse bajo fuertes controles autoritarios, como ocurrió en regímenes duales y excluyentes, como el mexicano, el boliviano, el salvadoreño y el ecuatoriano (Barba, 2003, p. 578-596; Mesa-Lago, 2001).

${ }^{37}$ Que no cierra el sistema público, pero sí lo reforma y lo convierte en uno de sus dos componentes: el sistema público paga una pensión básica, mientras el privado paga una pensión complementaria. Este tipo de modelo fue implantado en regímenes universalistas democratizados, como Argentina y Uruguay, donde la oposición política desempeñó un papel muy significativo (Barba, 2003, p. 578-596; Mesa-Lago, 2001)

${ }^{38}$ Que tampoco cierra el sistema público, el cual se mantiene como una alternativa al nuevo sistema privado, como ocurrió en regímenes duales y excluyentes como Colombia y Perú, que enfrentaron grandes resistencias a sus tentativas de implantación de sistemas sustitutivos (Barba, 2003, 578-596; Mesa-Lago, 2001).

${ }^{39}$ Para hacerlos más viables en términos financieros, como ocurrió en los casos de Costa Rica y Brasil (Barba,2003, p. 578-596; Mesa-Lago, 2001).

${ }^{40}$ En Brasil, en 1991, se estableció un programa de pensiones rurales para adultos mayores, conocida como Previdência Rural (PR) y, en 1993, un programa de pensiones urbanas para adultos mayores, denominado Beneficio de Prestação Continuada, que, en conjunto, protegen a 5.3 millones de personas (Barrientos, [et. al], 2003).

${ }^{41}$ Como el PNUD (1999), CEPAL (1999, 2001, 2001 a, 2002, 2004), Astorga y FitzGerald (1998) y Cominetti y Ruiz (1998). 
En el caso de los regímenes universalistas, los claro-oscuros de la década de los noventa son muy evidentes. Se dio una evidente mejora en términos de crecimiento económico y un comportamiento polarizado en términos de estabilidad macroeconómica. ${ }^{42}$ En los casos de Chile y Costa Rica, no se registraron modificaciones significativas en las tendencias históricas en materia de bienestar social: la precariedad laboral continuó aumentando, ${ }^{43}$ al igual que el desempleo y la concentración del ingreso que creció o se mantuvo alta, la pobreza disminuyó en términos relativos, pero aumentó en términos absolutos. En los casos de Argentina y Uruguay, como resultado de la reciente crisis económica, se experimentaron notables caídas del PIB per cápita y un repunte extraordinario de la pobreza, que exhibe graves fenómenos de vulnerabilidad social (Ver: Anexo 1).

Sin embargo, en este cluster, a pesar de las tendencias del empleo y de las reformas de los sistemas de pensiones, éstos mantuvieron altos niveles de cobertura. ${ }^{44} \mathrm{Y}$, a pesar de las tendencias en materia de pobreza y distribución del ingreso (Anexo 1), los índices relativos de vida ${ }^{45}$ al igual que los niveles de desarrollo humano, se mantuvieron como los mejores de toda la región. ${ }^{46}$ Así mismo, los indicadores de exclusión social se sostuvieron como los menores de América Lati$n^{47}$ (Barba, 2003, Cuadros 86, 89, 90).

${ }^{42}$ Mientras Chile y Costa Rica lograron una gran estabilidad, no fue así en los casos de Argentina y Uruguay después de la crisis reciente.

${ }^{43}$ De acuerdo con Stallings; Weller (2001, Cuadro 9) durante los años noventa en Chile el 21\% del empleo era por cuenta propia, mientras en Costa Rica lo era el 18.7\%

${ }^{44}$ Entre 1997 y 1998 en este cluster en promedio el 81\% de la fuerza laboral estaba asegurada (Mesa-Lago, 2001, Cuadro 3).

${ }^{45} \mathrm{Si}$ comparamos el nivel de bienestar de los países de este cluster con el de los Estados Unidos de América (tomado como 100) encontraremos que hacia 1970 el índice relativo de vida era de 76 y para 1995 era de 78 , lo que habla de una ligera reducción de la brecha (Astorga; FitzGerald, 1998, Cuadro IX.5).

${ }^{46}$ En 1997 los países que integran este cluster se encontraban ubicados entre las posiciones 34-45 del IDH (1) del PNUD, con valores promedio de ese índice de 0.825 (PNUD 1999).

${ }^{47}$ En 1997 el IPH-1 que refleja la proporción de la población afectada por una serie de privaciones clave en materia de longevidad, conocimientos, salud, desnutrición, e ingresos, para este cluster era en promedio de sólo el 4.3\% (Barba, 2003, Cuadro 90).
No obstante, como hemos visto, la situación de algunos de estos regímenes empezó a deteriorarse en la parte final del siglo XX y la crisis argentina hace albergar serias dudas sobre la sustentabilidad de las reformas estructurales realizadas y sobre la conveniencia de continuar acríticamente por la senda seguida hasta ahora (Anexo 1).

En el caso de los regímenes de bienestar duales, la precariedad laboral fue mucho mayor que en el caso anterior; los niveles de desempleo también fueron, en general, más altos. ${ }^{48} \mathrm{La}$ cobertura de la seguridad social, ahora reformada, siguió siendo también mucho más baja, lo que se tradujo en que amplios sectores de la población laboraran sin ningún tipo de protección social. ${ }^{49}$ Por otra parte, aunque el gasto social como porcentaje del PIB se recuperó, la brecha frente a los niveles de gasto social de los regímenes universalistas siguió siendo semejante a la de los años setenta. ${ }^{50} \mathrm{En}$ este cluster intermedio, progresaron los niveles de cobertura de los programas sectoriales de nivel primario en materia de educación y salud, hasta alcanzar casi la universalidad, empero la capacidad disminuyó conformé se avanza a otros niveles de servicio, donde la brecha respecto a los regímenes universalistas no redujo significativamente. ${ }^{51}$

En este caso, el PIB per cápita prácticamente no creció, ${ }^{52}$ lo que, sin duda, afectó negativamente la capacidad para reducir la pobreza y la pobreza extrema. Sin embargo, a pesar de que aumentó la concentración del ingreso a grados extremos ${ }^{53} \mathrm{y}$ de que los niveles de pobreza siguieron siendo muy altos, los índices relativos de vida mejoraron

${ }^{48}$ La excepción es México, pero eso también se relaciona con una forma distinta de medir el desempleo.

${ }^{49}$ Entre 1997 y 1998, en este cluster, en promedio el $36.0 \%$ de la fuerza laboral estaba asegurada, porcentaje muy inferior al de los regímenes universalistas (Mesa-Lago, 2001, Cuadro 3).

${ }^{50}$ En 1980-81, los regímenes universalistas destinaban en promedio el 16.4\% del PIB al gasto social, para 199899 destinaban el 19\%. En contraste, los regímenes duales destinaban respectivamente el $10.8 \%$ y el $13.4 \%$ del PIB (Cominetti; Ruiz, 1998, Cuadro 2; Barba, 2003, Cuadro 86).

${ }^{11}$ Ver: Urrutia, 1993; CEPAL, 2002; Barba, 2003, Cuadro 86

${ }^{52}$ En el caso de Venezuela, el PIB per cápita cayó de manera abrupta (Anexo I).

${ }^{53}$ La excepción es Colombia. 
y la brecha con los regímenes universalistas se redujo. ${ }^{54}$ No obstante, los índices de pobreza humana (IPH-1) fueron tres veces mayores que en el caso de dichos regímenes, ${ }^{55}$ lo que indica una proporción mucho mayor de población excluida de los servicios de salud, educación, nutrición y oportunidades de ingreso (Anexo 1).

Finalmente, en el caso de los regímenes excluyentes, los datos indican una situación social aún más difícil, esto a pesar de que los niveles de crecimiento del PIB per cápita fueron superiores a los alcanzados por los regímenes duales (Anexo 1). En este caso, aunque el desempleo fue comparativamente bajo, ${ }^{56}$ la precariedad laboral registrada fue muy alta ${ }^{57}$ y la cobertura de la seguridad social muy inferior a la de los casos previos. ${ }^{58} \mathrm{En}$ ese contexto, al igual que en un sector muy amplio de los regímenes duales, tener un empleo no garantizó salir de la pobreza, como lo demuestran los índices de pobreza prevalecientes en este cluster, que continuaron siendo los más elevados de América Latina. Esa situación se vió agravada por bajos niveles de gasto social y niveles muy altos de concentración del ingreso (Anexo 1).

Los niveles de vida también continuaron siendo los más reducidos de la región, a pesar de que, en las últimas décadas, creció la cobertura en materia de salud y educación y se incrementó la esperanza de vida. Esto es corroborado por los niveles de desarrollo humano que, si bien no pueden catalogarse como bajos, sí se ubicaron en-

${ }^{54}$ De acuerdo con Astorga y FitzGerald (1998, Cuadro IX.3), el índice relativo de vida de los regímenes duales era 10 puntos menor que el de los universalistas en 1970, mientras en 1995 era sólo 5 puntos menor (Ver: Barba, 2003, Cuadro 89).

${ }^{55}$ El valor promedio del IPH-1 para estos regímenes es $12.3 \%$, frente al $4.3 \%$ de los regímenes universalistas (PNUD, 1999).

${ }^{56}$ En 1999, en Bolivia, El Salvador, Honduras y Perú, usados como ejemplo, en promedio la tasa media anual del desempleo urbano fue de 7.4 (Ver: CEPAL, 2001a, Cuadro III.7; Barba, 2003, Cuadro 75).

${ }^{57}$ En este cluster, durante los años noventa, en promedio del $44 \%$ de los empleos fueron por cuenta propia (Stallings; Weller, 2001, Cuadro 9).

${ }^{58}$ En 1997-98, sólo el 12\% de la fuerza laboral estaba asegurada en Bolivia, sólo el 32\% en Perú, y, en muchos casos, no hay ni siquiera datos confiables (Mesa-Lago, 2001, Cuadro 3). tre los más bajos de los niveles medios. ${ }^{59}$ En perfecta armonía con este bajo perfil social, los indicadores de pobreza humana señalan que, en este cluster, los niveles de exclusión social eran considerablemente altos. ${ }^{60}$

\section{CONCLUSIONES}

La imagen general mostrada indica que, a inicios del siglo XXI en América Latina, el desarrollo social y el crecimiento económico no están articulados de una manera satisfactoria, y que queda pendiente, en la agenda social de la región, la construcción de sociedades más igualitarias e inclusivas, necesidad que implica retos diferenciales, dependiendo del tipo de régimen de bienestar desde donde se afronten.

Los regímenes universalistas, durante los años noventa, se caracterizaron por estrategias de crecimiento económico ${ }^{61}$ devinculadas de la generación de empleo. Esto implica que de continuar esta tendencia, cada vez más personas en edad laboral no tendrán garantizado un derecho civil fundamental: el derecho a un trabajo digno, remunerador y portador de derechos sociales. ${ }^{62} \mathrm{~A}$ esta trayectoria se suman evidencias muy claras de que el subempleo crece y la calidad del empleo se deteriora. Ello indica que estos regímenes, tradicionalmente vistos como los baluartes de los procesos de salarización a escala regional, están adquiriendo gradualmente las características de los regímenes duales, donde los derechos sociales tienden a desvincularse de los nuevos empleos.

Nuestra región puede caracterizarse por una situación paradójica: o logra crecimiento económico

${ }^{59}$ Por ejemplo: Bolivia ocupa el lugar 112, El Salvador el lugar 107, Nicaragua el 121 y Perú el 80 (PNUD 1999).

${ }^{60}$ En promedio, en Bolivia, El Salvador, Nicaragua y Perú, el valor del IPH-1 es de $21.5 \%$ de la población (PNUD 1999).

${ }^{61} \mathrm{O}$ estilos de crecimiento económico.

${ }^{62}$ Sólo Costa Rica logró una elasticidad empleo-producto razonable, pero, al mismo tiempo, se trata de un caso sui generis, ya que se distingue por una liberalización económica muy limitada y por adoptar un estilo de crecimiento que privilegia la creación de empleos no calificados. 
sin empleos, como ocurre en los regímenes universalistas, o vincula el crecimiento a la generación de empleos de baja calidad y alta informalidad, como ocurre en los regímenes duales y excluyentes. Por ello, en ambos casos, es indispensable incorporar en la agenda pública, como algo estratégico en términos sociales, el tema de la generación de empleos formales, acompañados de derechos sociales mínimos. En ese escenario, son imprescindibles tanto un rol activo del Estado, para favorecer inversiones productivas que generen empleos de calidad, como un cambio de óptica pública sobre el mercado laboral, el cual debe dejar de concebirse como un terreno sagrado, donde sólo las leyes del mercado deben imperar, y en cambio recuperarse como un espacio medular para la inclusión social. En ese sentido, tanto la formulación de políticas de activación laboral como de capacitación para el trabajo son cruciales, ya que, desde el punto de vista social, lo fundamental no puede ser la flexibilización laboral para reducir costos, sino evitar la formación de estamentos de baja calidad laboral, que impiden el ascenso social para amplios sectores.

Por otra parte, los efectos dramáticos de las crisis económicas sobre los niveles de pobreza durante los años noventa, emblematizados por las crisis mexicana y argentina, dos de los países más representativos en materia de liberalización económica, indican que, a escala regional, los procesos de ajuste son acompañados por una alta vulnerablidad social, que puede llevar no sólo a perder lo ganado en el terreno de la reducción de la pobreza, como ocurrió con la crisis mexicana de mediados de los años noventa, sino a que, en ese ámbito, regímenes universalistas alcancen perfiles sociales que se asemejan a los de los regímenes duales, como ocurrió con Argentina, o a que regimenes duales se acerquen a los perfiles de los regímenes excluyentes, como ocurrió con Venezuela.

Las crisis económicas y sus devastadores efectos sociales no sólo ponen de manifiesto las limitaciones de las redes mínimas de seguridad social, que no demuestran las capacidades contracíclicas que se les atribuyen, sino que evidencían que la decisión de cada gobierno de privilegiar un tipo de redes sobre otras es con frecuencia inadecuada frente a la diversidad de riesgos y retos sociales afrontados. Esto significa, por ejemplo, que los regímenes universalistas no sólo enfrentan problemas de desempleo, sino de empobrecimiento masivo; y que los regímenes duales y excluyentes no confrontan exclusivamente problemas de pobreza masiva, sino de falta de capacidades de la población para ascender socialmente o de desempleo o subempleo.

Así mismo, si bien los alcances de estrategias de focalización y descentralización pueden variar positiva y negativamente entre los distintos tipos de regímenes de bienestar regionales, en la tarea de complementar u obstaculizar la universalización de los servicios sociales, un tema central y pendiente es el de los mecanismos de selectividad y la permanencia de los programas sociales.

Durante los últimos veinte años, en América Latina, ha habido una proliferación de programas sociales, con distintos objetivos, ${ }^{63}$ con diferentes tipos de beneficiarios atendidos, empleando distintos tipos de estrategias de focalización. ${ }^{64}$ Sin embargo, prácticamente en ningún caso se ha considerado a los pobres como ciudadanos que cuentan con derecho a un mínimo de bienestar, ni se han establecido derechos particulares, ni titularidades para exigirlos. Esto ha derivado en una gran inestabilidad social, porque los programas, al no ser instituciones que materializan derechos sociales, han permitido la manipulación de los pobres con fines electorales o políticos, o han sido recortados en momentos críticos o eliminados por cuestiones políticas, prácticamente sin

${ }^{63}$ Que han fluctuado entre programas de emergencia para apagar estallidos sociales, programas diseñados para incrementar la gobernabilidad de los procesos de ajuste, desarrollo de fondos de inversión social o de programas de inversión en capital humano para enfrentar la reproducción intergeneracional de la pobreza, o redes mínimas de seguridad para enfrentar la vulnerabilidad social, han implicado transferencias monetarias condicionadas o incondicionadas, provisión directa o indirecta de servicios públicos, etc.

${ }^{64}$ Directa, indirecta y autofocalización. 
ninguna explicación, o siguen funcionado en un contexto donde los niveles mínimos de bienestar son un asunto dictaminado tecnocráticamente y no algo exigible al Estado, etc.

En ese escenario, algo esencial es determinar social y políticamente cuáles deberían ser los parámetros mínimos de bienestar a los que todos deberían tener derecho, cuáles son los mecanismos, normas, procedimientos y recursos disponibles y exigibles por cada ciudadano para alcanzar las metas establecidas y cuáles son los compromisos que cada uno debería asumir en este esfuerzo, tanto en términos presupuestales por parte de los no pobres, como en términos de obligaciones por parte de los pobres, para hacer uso de sus derechos.

Resulta claro que este proceso sería necesariamente desigual, dado que las brechas en términos de pobreza, exclusión, índices relativos de vida y de desarrollo humano, entre los distintos tipos de regímenes de bienestar, son muy marcadas, y los plazos para cumplir los compromisos dependerían de la voluntad pública para resolver democráticamente los problemas fiscales que el esfuerzo demandaría. Pero, en todo caso, este cambio de óptica daría una mayor certidumbre a los pobres y les garantizaría efectivamente un punto de partida común o recursos de emergencia exigibles, una ciudadanía y dignidad social para desarrollar sus propias expectativas, al margen de la manipulación y los vaivenes de la vida política y del comportamiento cíclico de la economía.

(Recebido para publicação em setembro de 2006) (Aceito em agosto de 2007)

\section{REFERÊNCIAS}

ALTIMIR, Oscar. Crecimiento, ajuste, distribución del ingreso y pobreza en América Latina. En: NÚÑ̃Z José (Ed.) Políticas de ajuste y pobreza. Falsos dilemas, verdaderos problemas. Washington, D.C.: Banco Interamericano de Desarrollo, 1995. p. 31-76.

ANNESLEY, Claire. UK social policy: between europeanization and americanization. Manchester: University of Manchester, 2001.
ASTORGA, Pablo; FITZGERALD, Valpy. Apéndice estadístico. En: THORP, Rosemary. Progreso, pobreza y exclusión. Una historia económica de América Latina en el Siglo XX. Washington D.C.: BID, Unión Europea, 1998.

BANCO MUNDIAL. World Development Report 1990.

Informe Sobre el Desarrollo Mundial 1994.

Informe Sobre el Desarrollo Mundial 1995. El mundo del trabajo en una economía integrada. 1995.

.Informe Sobre el Desarrollo Mundial 1997. El Es-

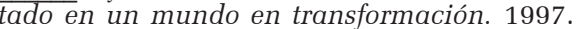

Informe Sobre el Desarrollo Mundial 2000/2001. $\overline{\text { Lucha }}$ contra la pobreza. 2001.

BARBA, Carlos El nuevo paradigma de bienestar residual y deslocalizado. Reforma de los regímenes de bienestar en la OCDE, América Latina y México. 2003. Tesis (Doctor en Ciencias Sociales) - Universidad de Guadalajara y el Centro de Investigaciones y Estudios Superiores en Antropología Social.

Los enfoques latinoamericanos sobre la política social: más allá del Consenso de Washington. En: Espiral. Estudios sobre Estado y Sociedad, v. 11, n. 31, p. 85-130, 2004.

Régimen de bienestar y reforma social en México. Santiago de Chile: ONU-CEPAL. 2004a (Serie las políticas sociales, 90).

(Coord.) Reforma social y ciudadanía social en América Latina durante los años noventa: Una perspectiva comparada. En: BARBA, Carlos (Coord.), Retos para la superación de la pobreza y la integración económica y social en América Latina. Buenos Aires: CLACSO, 2006. (en prensa).

BARBA, Carlos et al. Research horizons: poverty in Latin America. En: OYEN, Else et al. The polyscopic landscape of poverty research. "State of the art" in International Poverty Research. An overview and 6 in-depth studies. Bergen, Noruega: International Social Sciences Council and Comparative Research Programme on Poverty, 2005. p. 29-60.

BARRIENTOS, Armando et al. Non-Contributory pensions and poverty prevention. A comparative study of Brazil and South Africa. Reino Unido: Institute of Development and Policy Management y HelpAge International, 2003.

BANCO INTERAMERICANO DE DESARROLLO (BID). Estrategia para reducir la pobreza. Washington, 1997 Disponible en: http://www.iadb.org/sds/doc/904spa.pdf $>$.

Para salir de la pobreza. El enfoque del Banco Interamericano de Desarrollo para reducir la pobreza. Washington, 1998.

Protección Social para la Equidad y el Crecimiento. Washington D.C., 2000.

BEVERIDGE, W. H. Las bases de la seguridad social. México: Instituto Nacional de Salud Pública y Fondo de Cultura Económica, 1987 (Coleción biblioteca de la salud) .

BONOLI, George; TAYLOR-GOOBY, P. European welfare futures: towards a theory of etrenchment. Cambridge: Polity, 2000.

BOTTOMORE, Tom. Citizenship and social class, forty years on. En: MARSHALL, T.H; Citizenship and social class. Londres: Pluto Press, 1992. p. 55-95

BURKI, S.J.; EDWARDS, S. Latin America after Mexico: quickening the pace. Washington, D.C.: Banco Mundial, 1995 (Documento de trabajo).

CEPAL-Comisión Económica Para América Latina y el Caribe Transformación productiva con equidad. La tarea 
prioritaria del desarrollo de América Latina y el Caribe en los años noventa. Santiago de Chile: Naciones Unidas, 1990.

. Gasto social y equidad en América Latina. ( $L C / R$ 1235). Santiago de Chile, 1992.

. Panorama social de América Latina 1996. Santiago de Chile: Naciones Unidas, 1997.

. La brecha de la equidad. América Latina, el Caribe y la cumbre social. Santiago de Chile: Naciones Unidas, 1997a.

. Panorama social de América Latina 1998. Santiago de Chile: Naciones Unidas, 1999.

Equidad, desarrollo y ciudadanía. Resumen ejecutivo, 2000.

. Una década de luces y sombras: América Latina y el Caribe en los años noventa, 2001.

. Panorama social de América Latina 2000-2001. Santiago de Chile: Naciones Unidas, 2001a.

Anuario estadístico de América Latina y el Caribe 2001. Santiago de Chile: Naciones Unidas, 2002.

Panorama social de América Latina 2002-2003.

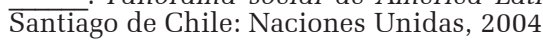

COMINETTI, Rossella; RUIZ, Gonzalo. Evaluación de gasto público social en América Latina: 1980-1995. Santiago de Chile: CEPAL, 1998.

DAHRENDORF, Ralf. The changing quality of citizenship. En: STEENBERGEN, Bart Von. The condition of citizenship. Londres: SAGE Publications, 1994. p. 20-35

DEACON, Bob. Socially responsible globalization: a challenge for the European Union. Helsinque: Ministerio Finlandés de Asuntos Sociales y Salud, 1999. Disponible en: <http//www.vn.fi/stm/english/tao/publicat/deacon/ contents.htm $>$.

EDWARDS, S. Crisis and reform in Latin America. From despair to hope. New York: Oxford University Press, 1995

ESPING-ANDERSEN, Gosta. The comparison of policy regimes: an introduction. En: ; MARTIN, Rein; RAINWATER, Lee (Eds.). Stagnation and renewal in social policy. The rise and fall of policy regimes. New York: M.E. Sharpe, Inc, 1987.

The three worlds of welfare capitalism. Cambridge, Gran Bretaña: Polity Press, 1990.

After the golden age. Welfare State dilemmas in global economy. En: ESPING-ANDERSEN, Gosta (Ed.) Welfare states in transition. National adaptations in global economy. Londres: Sage Publications, 1996, p.1-31

Social foundations of postindustrial economies Londres: Oxford University Press, 1999.

Reestructuración de la protección social. Nuevas estrategias de reforma en los países adelantados. En: RO-

$\therefore$ LANDO, Franco (Coord.) Sociología del desarrollo, políti $\rightarrow$ cas sociales y democracia. México: Siglo XXI /CEPAL, 2001, p.202-216

ESPING-ANDERSEN, Gosta; CORPI, Walter, El modelo escandinavo: del alivio a la pobreza a los sistema avanzados de protección. En: KLINSBERG, Bernardo. Pobreza un tema impostergable. México: Fondo de Cultura Económica, PNUD, CLAD, 1993. p. 369-420.

FALK, Richard. The making of global citizenship. En STEENBERGEN, Bart Von. The condition of citizenship. Londres: SAGE Publications, 1994. p. 127-140.

FILGUEIRA, Fernando. La nueva arena de las políticas sociales: vectores internacionales y mediación doméstica en la reforma del sector social en América Latina. En: PEREZ BALTODANO, Andrés. Globalización, ciudadanía y política social en América Latina: tensiones y contradicciones. Caracas: Nueva Sociedad, 1997. p. 67-96.

Tipos de welfare y reformas sociales en América atina Eficiencia, residualismo y ciudadanía estratificada. Ponencia presentada en el SEMINARIO A REFORMA DA ADMINISTRAÇÃO PÚBLICA NO BRASIL: possibilidades e obstáculos. Recife: Fundação Joaquim Nabuco, ago. 1998. 29p. Disponível em: <http://www.fundaj.gov.br/ docs/eg/Semi8.rtf>

FRANCO, Rolando. Grandes temas del desarrollo social en América Latina y el Caribe. En: SOJO, Carlos (Ed.) Desarrollo social en América Latina: temas y desafíos para las políticas públicas. San José de Costa Rica: Banco Mundial y FLACSO, 2002. p. 63-104.

GARCÍA, CANCLINI, Nestor. Consumidores y ciudadanos: conflictos multiculturales de la globalización. México: Editorial Grijalbo, 1995

GOODIN, Robert; HEADEY, Bruce; MUFFELS, Ruud HENK-JAN, Sirven. The real worlds of welfare capitalism. Cambridge, Reino Unido: Cambridge University Press. 1999.

GORDON, Sara. Desarrollo social y derechos de ciudadanía. En: SOJO, Carlos (Ed.) Desarrollo social en América Latina: temas y desafíos para las políticas públicas. San José de Costa Rica: Banco Mundial y FLACSO, 2002. p. 150214

GROPELLO, Emanuela di; COMINETTI, Rossella. La descentralización de la educación y la salud: un análisis comparativo de la experiencia latinoamericana. Santiago de Chile: CEPAL, 1998.

HICKS, Norman; WODON, Quentin. Are governments pro poor? A test based on targeted and social spending under booms busts. Washington D.C.: Banco Mundial, 1999.

. Protección social para los pobres en América Latina. Revista de la CEPAL, n. 73, abr., p. 95-116, 2001.

HABERMAS, Jürgen. Citizenship and nacional identity. En: STEENBERGEN, Bart Von. The condition of citizenship. Londres: SAGE Publications, 1994. p. 20-35. HILL, Michael; GLEN BRAMLEY. Analysing social policy. Oxford: Blackwell Publishers, 1986

Understanding social policy. Oxford: Blackwell Publishers, 1997.

HILLMERT, Steffen. Welfare state regimes and life-course patterns: an introduction. 2001. Disponível em: <http:// www.mpib-berlin.mpg.de/dok/full/e2001.0232/frames/ paper.htm>

HUBER, Evelyne; STEPHENS, John. The political economy of pension reform: Latin America in comparative perspective. Ponencia presentada en LASA 2000, Miami, mar., p. 16-18.

LAUTIER, Bruno. Las políticas sociales en América Latina: propuestas metodológicas para analizar el cambio que se está produciendo. Espiral: Estudios de Estado y Sociedad, [S.l.], v.8, n. 22, p.91-130, 2001.

MALLOY, James. Statecraft, política y crisis de la seguridad social. Una comparación de la América Latina y los Estados Unidos. En: MESA-LAGO, Carmelo. La crisis de la seguridad social y la atención a la salud. Experiencias y lecciones latinoamericanas. México: Fondo de Cultura Económica, 1986, p.29-68.

MARSHALL, T.H. Citizenship and social class. En MARSHALL, T.H; BOTTOMORE, Tom. Citizenship and social class. Londres: Pluto Press, p. 3-51(1950, 1992)

MESA LAGO, Carmelo. Changing social security in Latin America: towards alleviating the costs of economic reform. Londres: Lyenne Rienner Publisher, 1994. 
Privatización del sistema de pensiones en América Latina. En: FRANCO, Rolando (Coord.) Sociología del desarrollo, políticas sociales y democracia. México: Siglo XXI, CEPAL, 2001. p. 218-237.

PIERSON, Paul. Dismantling the welfare state? Regan, thatcher and the politics of retrenchment. Cambridge:University Press, 1994.

PROGRAMA DE LAS NACIONES UNIDAS PARA EL DESARROLLO (PNUD), Human development report 1999. Nueva York: Oxford University Press, 1999.

RACZYNSKI, Dagmar Focalización de programas sociales lecciones de la experiencia chilena. En: PIZARRO, Raczynski y Vidal (Ed.). Políticas económicas y sociales en el Chile democrático. Santiago de Chile: Cieplan/ UNICEF, 1995

ROMÁN, Luis Ignacio¿̇ué es el ajuste estructural? Racionalidad e irracionalidad de las políticas económicas de libre mercado. Guadalajara: ITESO/ SIMorelos, 1999.

SCHARPF, Fritz; SCHMIDT, Vivien. Welfare and work in the open economy. From vulnerability to competitiveness. Oxford: University Press, 2000.

SCHIERUP, Carl-Ulrik. Whither the social dimension? Citizenship, racialised exclusion and changing EU socia policy agendas. Ponencia presentada en la CONFERENCIA ENTRE ESTADOS Y MERCADOS. Lieja, 2001, p. 116. Disponible en: <http://www.ulg.ac.be/cedem/francais/ Tires/conf/Schierup_Carl.html>

SKOCPOL, Theda Americas incomplete welfare state: the limits of new deal reforms and the origins of the present crisis. En: ESPING-ANDERSEN, Costa; MARTIN, Rein; RAINWATER, Lee (Eds.) Stagnation and renewal in social policy. The rise and fall of policy regimes. New York: M.E. Sharpe, Inc., 1987, p. 35-58.

Social policy in the United States. Future possibilities in historical perspective. Princeton: Princeton University Press, 1995.
SOJO, Carlos; PÉREZ, Juan Pablo. Reinventar lo social en América Latina. En: (Ed.) Desarrollo Social en América Latina: Temas y desafíos para las políticas públicas. San José de Costa Rica: Banco Mundial y FLACSO, 2002. p. $13-62$

Dinámica sociopolítica y cultural de la exclusión social. En: ; GACITÚA, Estanislao; DAVIS, Shelton. Exclusión social y reducción de la pobreza en América Latina y el Caribe. San José, Costa Rica: FLACSO/ Banco Mundial, 2000. p. 51-8.

SKOCPOL, Theda. Social policy in the United States. Future possibilities in historical perspective. Princeton: Princeton University Press, 1995.

STEENBERGEN, Bart Von. The condition of citizenship. Londres: SAGE Publications, 1994.

Towards a global ecological citizen. En: STEENBERGEN, Bart Von. The condition of citizenship. Londres: SAGE Publications, 1994. p. 141-152.

TORFING, Jacobo. Politics, regulation and the modern welfare state. Londres: MacMillan Press, 1998.

TURNE, Bryan. Posmodern culture/modern citizens. En: STEENBERGEN, Bart Von. The condition of citizenship. Londres: SAGE Publications, 1994. p. 153-168

; ISIN, Engin. Handbook of citizenship studies. SAGE Publications, 2002.

VAN GUNSTEREN, Herman. Four Conceptions of Citizenship. En: STEENBERGEN, Bart Von. The condition of citizenship. Londres: SAGE Publications, 1994. p. 3648

WILLIAMSON, John (Ed.) Latin American Adjustment. How much has happened? IIE: 1990. 
AMÉRICA LATINA: regímes de bem-estar em transição durante os anos noventa

Carlos Barba

Este artigo analisa o andamento do processo de ajuste e de reforma social impulsionado pelas instituições financeiras internacionais e por numerosos governos na América Latina, especialmente nos anos 90. Apresenta as características do paradigma de bem-estar que chegou a ser hegemônico em escala regional e discute as conseqüências de aplicarem-se as mesmas receitas a realidades nacionais muito diversas. Para analisar a heterogeneidade regional, recorre ao conceito de "regime de bem-estar" e emprega uma tipologia que permite mostrar os efeitos diferenciais dos processos aqui abordados. O artigo aponta a necessidade do uso de um paradigma de bem-estar distinto para enfrentar os profundos desafios sociais postos aos países da região, ou seja, assinala a urgência de uma reforma da reforma.

PALAVRAS-CHAVE: regimes de bem-estar, pobreza, políticas sociais, transição social, América Latina.

\section{LATIN AMERICA: welfare system in transition during the nineties}

\section{Carlos Barba}

This paper analyzes the course of the adjustment and social reform processes impelled by international financial institutions and numerous governments in Latin America, especially in the nineties. It presents the characteristics of the welfare paradigm that achieved hegemony in a regional scale and discusses the consequences of applying the same recipes to very diverse national realities. To analyze the regional heterogeneity, it falls back upon the concept of "welfare system" and uses a typology that shows the differential effects of the processes approached here. The paper points the need of the use of a different welfare paradigm to face the deep social challenges put to the countries of the area, in other words, it marks the urgency of a reform of the reform.

KEYwORDS: welfare system, poverty, social policies, social transition, Latin America.

\section{AMÉRIQUE LATINE: les régimes de bien-être en phase de transition au cours des années quatre-vingt-dix}

\section{Carlos Barba}

Cet article analyse la progression du processus d'ajustement et de réforme sociale provoquée par les institutions financières internationales et par de nombreux gouvernements en Amérique Latine, tout spécialement au cours des années 90. Les caractéristiques du paradigme de bienêtre dont le caractère hégémonique a atteint une échelle régionale y sont présentées et les conséquences dues au fait d'appliquer les mêmes formules à des réalités nationales très diverses y sont discutées. Afin d'analyser l'hétérogénéité régionale, on a recours au concept de "régime de bien-être" et on utilise une typologie qui permet de montrer les effets différentiels des processus qui ont été abordés. Dans cet article le besoin d'utiliser un paradigme de bien-être différent pour affronter les profonds défis sociaux auxquels sont confrontés les pays de la région est mis en évidence, c'est-àdire qu'on y montre l'urgence d'une réforme de la réforme.

Mots-CLÉs: régimes de bien-être, pauvreté, politiques sociales, transition sociale, Amérique Latine.

Carlos Barba Solano. Sociólogo, Professor-investigador Titular da Universidade de Guadalajara, no doutorado e mestrado em ciências sociales, e na licenciatura em sociologia. Membro do Sistema Nacional de Investigadores. Doutor em Ciências Sociais pela Universidade de Guadalajara, mestre pela Universidad de WisconsinMadison e licenciado em relações internacionais por El Colegio de México. É diretor da revista Espiral: Estudios de Estado y Sociedad, que integra o Índice Mexicano de Revistas Científicas do Consejo Nacional de Ciencia y Tología e coordena o Grupo de Pesquisa "Pobreza y Políticas Sociales" do CLACSO. Publicou inúmeros artigos, capítulos e livros, entre eles:¿Reducir la pobreza o construir ciudadanía social para todos? América Latina: regímenes de bienestar en transición al iniciar el siglo XXI. Guadalajara/MX: Universidad de GuadalaMéxico e na América Latina. 